\title{
ZUR HERKUNFT \\ DES ENGLISCHEN GERUNDIUMS.
}

Auf Curmes obige ausführungen habe ich folgendes zu erwidern.

Dafs in interlinearübersetzungen und übertragungen ähnlicher art gerundien vorkommen, ist bekannt; ich habe selbst deren mehrere nachgewiesen. Es ist aber deutlich erkennbar, dafs sie sämtlich ganz sklavische und mechanische nachbildungen von lateinischen gerundien sind und ebensowenig beweisen können, dafs sie zu dem eigentlichen körper ihrer sprache gehören, wie die genau entsprechenden nachbildungen lateinischer gerundien in althochdeutschen schriften ähnlicher art. ${ }^{1)}$ Bezüglich aller dieser vorkommnisse ist also Curmes

1) Der einzige beleg, der für eine freie einheimische verwendung eines gerundiums zu sprechen scheint, ist der von Curme auf s. 495 angefuihrte ' $h i$ (sic!) sylfe to clansunga for wordunga dare godcundan orowounga and aristes'. Aber auch er hält einer genauen prüfung nicht stand: wäre claensunga ein gerund, so mülste hi sylfe (wie meine listen $35 \mathrm{ff}$. beweisen) zwischen ihm und der dazu gehörigen präposition stehen. $\mathrm{Da}$ (s es dies nicht tut, sondern die übliche stellung des dativus commodi einnimmt, ist an sich schon ein genügender beweis, dafs wir es hier nicht mit einem gerund sondern mit einem verbalsubstantive zu tun haben; im urtext Aelfrics wird jedenfalls nicht hi sylfe, mit grofser wahrscheinlichkeit aber him sylfum gestanden haben, eine störung, welche sich durch schreibflüchtigkeit nicht allzu schwer erklärt: zunächst irrtümlich $h i$ statt $h i$, dann in bewufster angleichning an erstere akkusativform sylfe statt sylfum. Ich will noch bemerken, dals nicht nur diese stelle, sondern das ganze stück den eindruck einer ganz ungeschickten übersetzung (natürlich aus dem Lat.) macht. Es böte sich somit noch die möglichkeit einer anderen erklärung dieser auffälligen konstruktion. Ich kann hier der sache nicht weiter nachgehen, auf jeden fall ist hi sylfe to clansunga ein ganz barbarisches Altenglisch. 
ausdruck "establisht" (p. 495 u. ö.) durchaus nicht am platze, sondern sein ausdruck 'attempts'. Auch die von Curme p. $497 \mathrm{f}$. aus der deutschen humanistenzeit angeführten belege sind solche 'attempts"; nichts weiter. Trotz all dieser versuche, die sich zwanglos aus der allgemeinen zeitrichtung erklären und mit ihr verschwinden, 1) hat das Deutsche sich kein gerundium erobert. Es hat an seinem verbalsubstantive festgehalten. Wie es hat kommen können, dals das Englische aus seinem verbalsubstantive ein gerundium herausbildete, das Deutsche aber nicht, das ist der punkt, auf den es ankommt, das ist das rätsel, das es zu lösen gilt. C. hat aber dieses rätsel nicht gelöst und zwar einfach deshalb nicht, weil mit den mitteln, die er anwendet, es gar nicht gelöst werden kann. Dals aus dem zusammenschreiben und trennen der teile der komposita in den alten MSS. keine auch nur einigermafsen sicheren schlüsse auf die syntaktische geltung ihrer beziehung auf einander zu ziehen sind, habe ich schon früher ausgeführt. $\mathrm{Zu}$ sagen, dals der erste teil einer solchen getrennt geschriebenen wortgruppe als objekt empfunden wurde, ist also gänzlich unzulässig. Curmes zweites, und wie es scheint, hauptmittel, des obenbezeichneten rätsels lösung herbeizuführen, ist noch weniger einwandfrei. Dies mittel besteht in der nach seiner ansicht im Mittelenglischen sich ändernden wortgruppenbetonung mit der tendenz der stärkeren hervorhebung des zweiten gruppenteiles. Hiergegen sprechen jedoch zwei tatsachen. Die erste, darin bestehend, dals aus den im Deutschen vorhandenen gleichen verhältnissen sich kein gerund entwickelte, wird von C. selbst bemerkt, von ihm aber durch den hinweis beseitigt, dals die neben jenen gruppen bestehenden anderen gruppen, wie 'feuer machen' und 'feuer gemacht', eine derartige entwickelung (um deutlicher zu sein: umstellung!) verhindert hätten. Hiergegen ist aber einzuwenden, dafs diese letzteren gruppen gar keine gruppen im

1) Hieranf ist zu achten. Es wird C. nicht möglich sein, eine verbindung jener (lateinisch-deutschen) gerundien der humanistenzeit mit den aus englischen einflüssen entstandenen gerundien der neuzeit herzustellen. Den gleichen englischen einflüssen entstammt ubrigens auch der deutsche betonte artikel: 'der feldherr' $=$ der bedeutendste feldherr, sowie fügungen wie 'eine formelle beruhrung mit dem, ja vielleicht ein aufgehen in dem, partizipium', u. a. m. 
sinne der ersteren sind, und dafs, wenn sie es wären, doch die neben ihnen bestehenden noch viel gebräuchlicheren 'ich mache feuer', 'ich machte feuer', ihre hemmende wirkung aufgehoben haben würden.

Die zweite tatsache, die gegen C.'s erklärungsweise spricht, besteht darin, dals jener nebenton auf dem zweiten kompositionsgliede in altenglischer zeit offenbar noch viel stärker war als in mittelenglischer, in welcher jener nebenton klärlich, verglichen mit der tonstärke im Altenglischen, nur eine mittlere stärke gehabt haben kann, dergestalt dafs der, welcher diese theorie Curme's anerkennt, ein reicheres auftreten des vollentwickelten gerundiums schon im Altenglischen zu erwarten berechtigt wäre. Dals ein solches in diesem umfange vorhanden, ist nun freilich die ansicht Curme's. Ich glaube aber schon genügend und des öfteren gezeigt zu haben, dafs C.'s belege durchaus nicht im stande sind, diese seine meinung $\mathrm{zu}$ stützen. Beian sei erwähnt, dafs im allgemeinen die nachstellung der objekte, sowie anderer adverbieller bestimmungen mit einer etwaigen tonverschiebung nichts zu tun hat, sondern als notwendige folge aus dem verluste der flexion sich ergab, vgl. Pauls Grdr., Syntax $§ 188$ und Keilmann, Dat. und Akk. beim Verbum, Diss. Giefsen 1909.

'Trotz allen bemühungen Curmes bleibt eben die tatsache bestehen, dafs es abgesehen von jenen wenigen und ungeschickten nachbildungen lateinischer gerundien ein altenglisches gerundium nicht gab. DaIs die englische sprache später ein solches entwickelte und damit einen den übrigen germanischen sprachen fremden abweg einschlug, beweist eben von vornherein, dals sie hierin anderen einflüssen folgte als denen, welchen die letzteren im laufe ihrer entwickelung ausgesetzt waren, und dals sie hierin auf keinen fall in ihr selbst ruhenden tendenzen folgte; denn wäre sie dies, dann wäre es ein wunder, wenn gleiche tendenzen in den übrigen schwestersprachen nicht den gleichen erfolg gehabt hätten.

Curme sagt auf seite 497 'Language development must be interpreted as inner growth until foren influence is absolutely manifest'.

Es wundert mich, dals C. dies mir sagt, mir, der ich genau diese ansicht an den verschiedensten stellen meiner schriften betont und vertreten habe. Es ist dies so genau 
meine ansicht, dals der zitierte satz mir auf den ersten blick so vorkam, als sei er von mir abgeschrieben. Was nun den vorliegenden fall der entstehung des englischen gerunds angeht, so ist es mir eben nicht möglich gewesen, eine entwickelung von innen heraus nachzuweisen, trotz allen bemühungen. Und ebensowenig ist dies $\mathrm{C}$. möglich gewesen, wie er sicher selbst erkannt haben würde, wenn er seine argumente etwas dichter unter die lupe genommen hätte.

Und was nun übrig bleibt, ist eben die annahme eines eindringens von aufsen. In der tat, es wäre an der zeit, wenn Curme nach seinen wiederholt verunglückten versuchen, ein dem altenglischen sprachkörper angehöriges gerund nachzuweisen, sich nun endlich die frage vorlegte, ob eine solche annahme des eindringens von aufsen, wenn auch nur in diesem einen falle, der wahrscheinlichkeit gar so fern liege. Freilich eine solche frage auch nur ins auge zu fassen, ist ihm äufserst zuwider. Das erkennen wir aus seiner äufserung auf seite 497 'The riter is fighting for a cherisht principl' etc. C. sollte sich aber doch klar machen, dals er mit diesen worten wissenschaftlichen materien gegenüber eine anschauung verrät, welche seitens der gelehrten keine ungeteilte billigung erfahren dürfte. Oder glaubt $C$. etwa gar, ich 'cherishe' auch ein 'principl'? Das dem seinen entgegengesetzte? Dann hat er sich gründlich geirrt. Ich habe und hatte von jeher nur das eine ziel: den weg der entwickelung aufzuklären; wohin dieser weg mich führte, war mir völlig gleichgiltig. Das überliefs ich den tatsachen, den mir zur verfügung stehenden belegen. Oft genug habe ich bedauert, vermutungsweise ein romanisches etymon setzen zu müssen aus mangel an germanischen belegen. Auf jeden fall also: eine voreingenommene stellung, wie sie sich in den eben zitierten englischen worten verrät, (als prinzipienreiten bezeichnen wir es in landlänfiger weise) muls den forscher ja geradezu verleiten, vereinzelt auftauchende erscheinungen als typisch zu überschätzen und, im allgemeinen, apriorische wahrscheinlichkeiten ja selbstverständlichkeiten zu milsachten.

Die eben genannten apriorischen selbstverständlichkeiten spielen nun aber gerade in den uns vorliegenden fragen eine besonders wichtige rolle. In ihnen hat die geschichte und die psychologie das letzte und entscheidende wort. Ich habe 
diesen gegenstand an verschiedenen stellen meiner schriften des genaueren erörtert, habe es also nicht nötig, mich hier zu wiederholen. Nur zwei einfache fragen möchte ich in verbindung damit herrn $\mathrm{C}$. vorliegen:

Erstens: Ist C. bereit, im falle der innigen berührung der einen sprache mit einer anderen, die gegenseitige syntaktische beeinflussung derselben in abrede zu stellen?

Zweitens: Ist C. gesonnen, eine derartige gegenseitige beeinflussung für den fall der mittelenglischen und normannisch-französischen sprache abzuleugnen?

Ist er es nicht, so hat er aus den vergeblichen versuchen (die angestellt $\mathrm{zu}$ haben, ihm niemand verübeln wird; ich am wenigsten!), eine englische spracherscheinung als "inneres gewächs" zu erweisen, die konsequenz zu ziehen, die jeder nicht voreingenommene gelehrte ziehen mufs, und die auch ich schon vor längerer zeit zu ziehen mich genötigt fühlte.

Ist er es aber - und sein eben zitiertes 'principl' sowie seine eigenartige prophezeiung in den Engl. St. 45 p. $377^{1}$ ) scheint darauf hinzuweisen, dals er in der tat zu einer derartigen ableugnung bereit ist, dann verweise ich ihn hiermit auf die in der einleitung zu meinem 'Indefinitum' angezogenen untersuchungen Windischs und Schuchardts. Mag er sich mit jenen gelehrten auseinandersetzen; der fall ist dann für mich erledigt.

Auf jeden fall kann es nicht scharf genug betont werden, dafs die nichtberücksichtigung oder auch nur die ungenügende berücksichtigung des romanischen einflusses auf die englische syntax einen schweren methodischen fehler darstellt. Einen solchen fehler $z u$ begehen, wird sich der fachmann, wird sich jeder gelehrte schwer hüten.

Zum schlusse sei bemerkt, dals in meinem anschreiben "Zur Geschichte des englischen Gerundiums" sowie in meiner

1) Zu Kellners äufserung "This construction is due to the French Original" bemerkt C. "This remark belongs to a long list that ought to be stricken out of our learned literature. They will soon appear very queer to readers". Auf grund der obenberührten historischen und psychologischen verhältnisse lälst sich vermuten, dafs trotz vereinzelten abstrichen jene liste immer länger werden wird, und dafs im gegenteil die versuche Curmes und seiner gesinnungsgenossen recht bald 'den lesern sehr seltsam vorkommen werden'. 
abhandlung "Die Entwickelung des englischen Gerundiums" doch sehr viel mehr steht - vieles namentlich, was in nächster beziehung zu dem hier angeschnittenen thema steht - als das, was herr Curme in seiner vorstehenden erwiderung zu widerlegen versucht hat.

Halle a/S., im September. E. Einenkel.

\section{BERICHTIGUNG.}

In meinem im vorigen hefte enthaltenen aufsatze "Nochmals zur fügung "A good one'" ist auf p. 209 z. 2 v. u. der name des von mir zitierten kollegen während des ausdruckes leider arg verstümmelt worden. Gemeint ist der namentlich in meinem spezialgebiete tätige gelehrte, herr Trampe Bødtker, wie der eingeweihte wohl auch schon aus den druckresten vermutet haben wird.

E. 